\title{
Three-dimensional genome architecture and emerging technologies: looping in disease
}

\author{
Arpit Mishra and R. David Hawkins*
}

\begin{abstract}
Genome compaction is a universal feature of cells and has emerged as a global regulator of gene expression. Compaction is maintained by a multitude of architectural proteins, long non-coding RNAs (IncRNAs), and regulatory DNA. Each component comprises interlinked regulatory circuits that organize the genome in three-dimensional (3D) space to manage gene expression. In this review, we update the current state of 3D genome catalogues and focus on how recent technological advances in 3D genomics are leading to an enhanced understanding of disease mechanisms. We highlight the use of genome-wide chromatin conformation capture ( $\mathrm{Hi}-\mathrm{C})$ coupled with oligonucleotide capture technology (capture $\mathrm{Hi}-\mathrm{C}$ ) to map interactions between gene promoters and distal regulatory elements such as enhancers that are enriched for disease variants from genome-wide association studies (GWASs). We discuss how aberrations in architectural units are associated with various pathological outcomes, and explore how recent advances in genome and epigenome editing show great promise for a systematic understanding of complex genetic disorders. Our growing understanding of 3D genome architecture-coupled with the ability to engineer changes in it-may create novel therapeutic opportunities.
\end{abstract}

\section{Background}

Chromosomal organization and compaction is an evolutionarily conserved feature. Large genomes need to be condensed into the minute 3D space of the nucleus in a systematic manner in order to retain functional capacity to interact with the gene regulatory machinery. Such a robust yet dynamic looping architecture facilitates finetuning of gene expression by mediating the contacts between distantly located cis-regulatory elements. Hence, spatial DNA organization performs a secondary role as a global regulator of gene expression. The 3D architecture of DNA is hierarchical in nature (Fig. 1). The fundamental architectural units develop from interactions of DNA and histone octamers in the form of nucleosomes, which leads to the formation of chromatin fibers. Chromatin fibers are further looped and facilitate regulatory interactions by forming insulated neighborhoods of regulatory loops, where multiple regulatory loops assemble to form chromosomal domains or

\footnotetext{
*Correspondence: rdhawk@uw.edu

Division of Medical Genetics, Department of Medicine, Department of Genome Sciences, Institute for Stem Cell and Regenerative Medicine, University of Washington School of Medicine, Seattle, WA 98195-5065, USA
}

topologically associated domains (TADs), on the scale of 500 kilobases $(\mathrm{kb})$ to 1 megabase $(\mathrm{Mb})$ [1-3]. The currently favored model suggests that TADs are formed by looping long stretches of DNA via anchor proteins such as the CCCTC-binding factor (CTCF)-cohesin complex [4-6]. Intra-TAD looping, including regulatory loops, primarily facilitates dynamic gene expression, while a minor fraction of gene regulatory looping also crosses TAD boundaries, known as inter-TAD regulatory loops (Fig. 1) $[7,8]$. TAD sizes are organized for enhancer-togene target functionality, and physical insulation of interactions within TADs indicates that regulatory functionality is further optimized at the sub-TAD level [9].

Associations of similar TADs form the next level of organization, known as chromosomal compartments. Referred to as A/B compartments, $\mathrm{A}$ is associated with the open euchromatin of transcriptionally active states and is found internally in the nucleus, while B is associated with closed chromatin that lacks significant histone modification enrichment and transcriptionally silent states, found at the nuclear periphery [6]. TAD organization also follows a hierarchical tree-like structure 


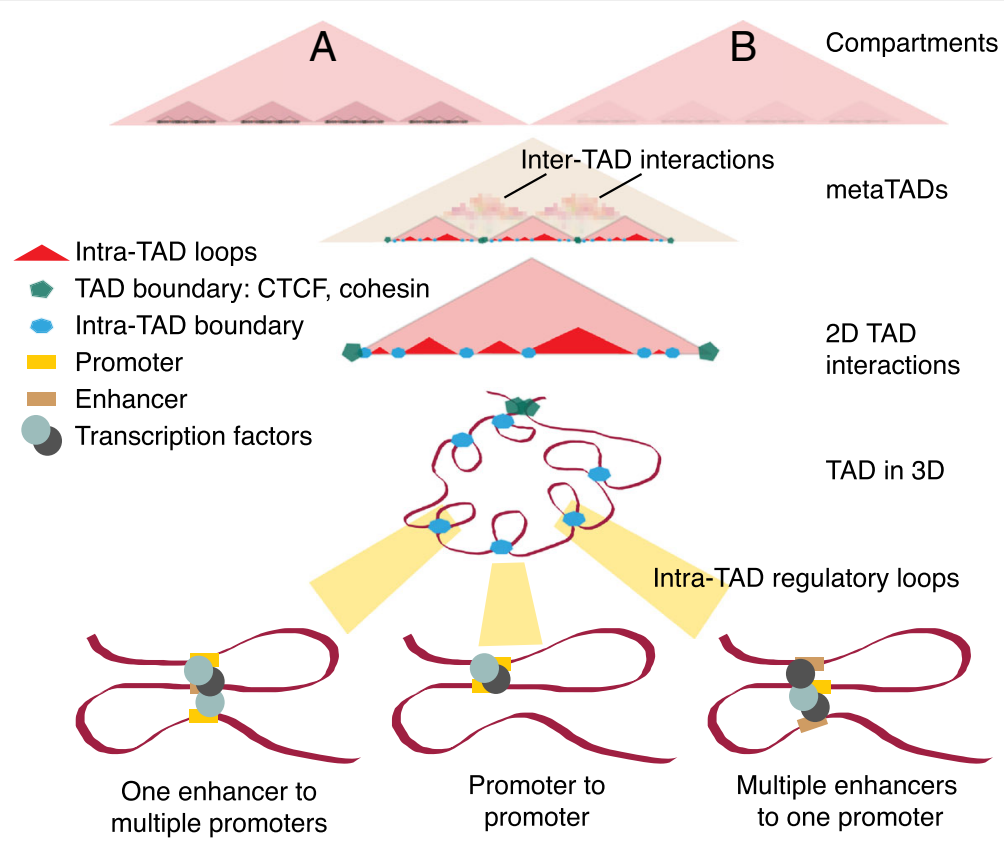

Fig. 1 Hierarchical chromatin organization. Top tier: higher-order compartments A and B, where A is an active compartment and B is an inactive or densely packed compartment (beige-colored top-most triangles). Moving downward, topologically associated domains (TADs) are organized into increasingly higher-resolution structures. Second tier. representative metaTAD structure (gray-colored triangle), where many TADs together form one metaTAD. Inter-TAD interactions, while more sparse, can be detected. Third tier. TADs (light pink triangle) consist of numerous intra-TAD regulatory loops (small red triangles in TADs). These regulatory loops are major governing factors for differential transcriptional output. In tiers 1-3, triangles represent higher-frequency contacts of the three-dimensional (3D) genome shown in two dimensions (2D). Tier four illustrates how a TAD may look in 3D, comprising intra-TAD regulatory loops. Representative examples of regulatory loops are also shown: one enhancer to multiple promoter interactions, promoter-promoter interactions, and multiple enhancers to one promoter interactions. TAD boundaries are marked by the CTCF-cohesin complex (green pentagon). Intra-TAD elements likely consist of different transcription factors (light green circles) and long non-coding RNA (dark gray circles)

$[9,10]$. TADs interact to form metaTADs; however, the interacting TADs are not always nearest neighbors, which suggests that hierarchical complexity rather than linear distance governs chromosomal organization [10]. At the highest order of organization each chromosome occupies a distinct chromosomal territory [11-13].

To sustain dynamic genome architectural changes cells deploy multiple tools. Major architectural proteins include CTCF, cohesin, lamins, the Mediator complex, and transcription factors (TFs). CTCF, an 11-zinc-finger-domain DNA-binding protein, is one of the most studied architectural proteins [14]. Approximately $15 \%$ of CTCF-binding sites are found at TAD boundaries, and most other binding sites are involved in intra-TAD regulatory loop interactions [15]. CTCF binds at CpGcontaining motifs, and DNA methylation at these sites can abrogate CTCF binding [16, 17]. Cohesin, which was first shown to facilitate sister chromatid cohesion $[18,19]$, aids looping through interactions with other architectural proteins and protein complexes such as CTCF and Mediators. In the absence of cohesin, TADs remain intact, but their overall packing is affected, resulting in increased inter-TAD interactions and reduced intra-TAD interactions $[13,20]$. The Mediator complex helps form the pre-initiation complex at active genes through its interactions with transcriptional machinery bound to cis-regulatory elements both proximal and distal to genes, such as promoters and enhancer elements, respectively [19]. This form of regulatory looping brings together enhancers and target promoters, which can be kilobases to megabases apart in the linear DNA sequence (Fig. 1).

In addition to TADs often being found internally in nuclear compartments, there is another component to chromosomal architecture that is near the nuclear periphery or nuclear lamina. These chromosomal architectural units are known as lamina-associated domains (LADs). LADs mainly consist of gene-depleted regions and are part of $B$ compartments $[1,6,10]$. They are mainly associated with lamin $\mathrm{B}$, lamin $\mathrm{A}$, and its alternative spliced product lamin C [21]. Finally, lncRNAs serve key roles in mediating chromosomal architecture (for review see [22]), as illustrated by one of the best characterized lncRNAs, XIST, which regulates the compaction of the inactive $\mathrm{X}$ chromosome by creating one compact mega domain and preventing TAD formation. The interplay of these factors gives dynamicity to the genome and influences the position of the mutational landscape. 
Defining genome architectural mechanisms of diseases will provide novel avenues for disease treatment and management. An advanced understanding of the human genome sequence and GWASs has led to the discovery that the majority of disease-associated mutations or genomic rearrangements lie in gene-desert (non-coding) regions of the genome. Unlike pathogenic mutations in coding regions, the molecular mechanisms of disease for these kinds of genomic aberrations cannot be as easily connected to underlying target genes. A genome architectural context for these variations may provide an understanding of how non-coding mutations influence pathology by altering cis-regulatory sequences such as enhancers, silencers, and insulators. These local or global changes in DNA topology may explain molecular mechanisms for many disorders, including cancer and developmental disorders.

The field of 3D genome organization is rapidly progressing and is already revealing the $3 \mathrm{D}$ structure to have a role in disease biology [13, 23, 24]. In this review, we provide a brief overview of recent technical advances and a further update on how 3D genomics is impacting our understanding of disease. Techniques such as singlecell $\mathrm{Hi}-\mathrm{C}$, capture $\mathrm{Hi}-\mathrm{C}(\mathrm{CHi}-\mathrm{C}), \mathrm{Hi}-\mathrm{C}$ chromatin immunoprecipitation (HiChIP), and proximity ligation-assisted chromatin immunoprecipitation followed by sequencing (PLAC-seq), when combined with GWASs and other omic, microscopy, and CRISPR-based approaches, are helping elucidate the mysteries of chromosomal organization-mediated gene regulation (Table 1). Moreover, we have highlighted the importance of publicly available 3D genome maps for linking regulatory mutations to target genes, and how disease phenotypes mediated by architectural changes can be reconstructed in model systems using genome editing to gauge underlying mechanisms. These novel combinatorial methodologies have already successfully identified pathomechanisms for various diseases.

Table 1 Commonly used terminologies

\begin{tabular}{|c|c|}
\hline Terminology & Definition \\
\hline Euchromatin & $\begin{array}{l}\text { Chromatin that contains loosely packed nucleosomes. Usually represents transcriptionally } \\
\text { active sites in the genome, including regulatory elements }\end{array}$ \\
\hline Heterochromatin & $\begin{array}{l}\text { Chromatin that is densely packed with nucleosomes. Usually represents transcriptionally } \\
\text { silent site in the genome }\end{array}$ \\
\hline DNase I hypersensitive sites (DHSs) & $\begin{array}{l}\text { Nucleosome-free regions of chromatin that are mostly found at enhancers and promoters. } \\
\text { Largely indicative of transcription factor binding }\end{array}$ \\
\hline Enhancer elements & $\begin{array}{l}\text { Enhancers are sequences of DNA that enhance gene expression by being bound by } \\
\text { transcription factors and looping to interact with gene promoters. These elements are } \\
\text { located on the same chromosome (cis-regulatory) and can be near promoters or } \\
\text { megabases away }\end{array}$ \\
\hline Super-enhancer & $\begin{array}{l}\text { Group of multiple enhancers located within } 12 \mathrm{~kb} \text { of each other, which are bound by an } \\
\text { array of transcription factors and marked by acetylation }\end{array}$ \\
\hline Temp enhancer & $\begin{array}{l}\text { A novel class of cis-regulatory elements whose disruption leads to temporary loss of target } \\
\text { gene expression, which is eventually regained }\end{array}$ \\
\hline Human-gained enhancer & $\begin{array}{l}\text { Putative novel enhancer-like elements gained in the human lineage, discovered from } \\
\text { brain } \mathrm{Hi}-\mathrm{C} \text { data }\end{array}$ \\
\hline Purifying selection & Negative selection in which deleterious alleles are selectively removed through evolution \\
\hline Gene desert & $\begin{array}{l}\text { Large genomic regions that are devoid of genes, but may harbor many disease-causing } \\
\text { variants and distal regulatory elements }\end{array}$ \\
\hline Promoter interacting regions (PIRs) & $\begin{array}{l}\text { PIRs are broadly defined as distal regulatory elements interacting with promoters via } \\
\text { looping interactions }\end{array}$ \\
\hline Frequently interacting regions (FIREs) & $\begin{array}{l}\text { FIREs are regional groups of putative enhancer-like elements that interact with each other } \\
\text { and many promoters }\end{array}$ \\
\hline Population average ensemble structure & $\begin{array}{l}\text { During } \mathrm{Hi}-\mathrm{C} \text { experiments in bulk, cells are present in multiple growth stages; thus, they } \\
\text { exhibit multiple } 3 \mathrm{D} \text { architectural landscapes. In bulk Hi-C, different architectural landscapes } \\
\text { are captured and this is called population average ensemble structure }\end{array}$ \\
\hline Haplotype phasing & $\begin{array}{l}\text { Deciphering haplotype block structures for polymorphic sites using genotype data. This is } \\
\text { traditionally done computationally to determine if variants are on the same allele. Hi-C } \\
\text { provides an experimental means of determining if variants reside on the same allele }\end{array}$ \\
\hline Combinatorial indexing & $\begin{array}{l}\text { Method that tags DNA within intact nuclei in each cell with successive rounds } \\
\text { (combinatorial) of nucleic acid barcodes for adapting to different genomics application } \\
\text { such as transcriptomics, Hi-C and chromatin accessibility for single-cell studies, without the } \\
\text { need for isolating single cells physically }\end{array}$ \\
\hline
\end{tabular}




\section{Approaches to studying disease and 3D genome architecture}

Approaches to understanding 3D genome architecture can be divided into two major categories. The first comprises microscopy and fluorescent in situ hybridization (FISH), methods that allow visualization of looping interactions. With the advent of super-resolution and cryo-electron microscopy, resolution limits have improved such that 11-angstrom structures for 30nanometer fibers can be achieved [25]. The second category covers chromosomal conformation capture (3C)-based approaches, which leverage proximity ligation to "capture" looping interactions $[13,26])$. There are now a number of 3C-based methods (for recent reviews see [26-28]; Table 2). The most relevant to this review is $\mathrm{Hi}-\mathrm{C}$ and its derivatives, which in principle can capture all interactions genome-wide, connect cis-regulatory elements harboring disease variants with their target genes, and provide insight on large structural rearrangements in the genome.

In Table 2, we briefly summarize the most suitable applications and limitations of genome architectural methods (for a detailed review see [29-31]) and list suitable computational pipelines for analysis of these genome architectural data.

$\mathrm{Hi}$-C-based approaches are at the forefront of guiding our understanding of TAD-level organization and loop formations. HiC-based 3D maps of the genome continue to improve in resolution. High-resolution architectural maps for nine different cell types [6] further reduced the average size of TAD organization to around 185$200 \mathrm{~kb}-\mathrm{a}$ substantial reduction from early studies $[1,2]$. Improving the resolution of 3D maps provides a framework for fine-mapping interactions of novel distal disease variants and their target genes, which could be of therapeutic interest.

\section{$\mathrm{CHi}-\mathrm{C}$ and similar directed $\mathrm{Hi}-\mathrm{C}$ approaches}

Deconvolution of the genetic basis of diseases requires high-resolution interaction maps for all genic elements. For now, reliable identification of intra-TAD interactions-such as regulatory loops-from $\mathrm{Hi}-\mathrm{C}$ data remains a challenge due to the complexity of $\mathrm{Hi}-\mathrm{C}$ libraries and the substantial cost for the sequence depth required to achieve statistically significant interactions. While targeted locus amplification (TLA) and targeted chromatin capture (T2C) techniques (Table 2) promise to provide cis-regulatory information for a limited subset of clinically relevant loci at a substantially reduced cost, $\mathrm{CHi}-\mathrm{C}$ and subsequent variations for improving throughput were developed to enrich for regions of interest across the genome from complex $\mathrm{Hi}-\mathrm{C}$ libraries by utilizing specific probes against preselected bait regions in a manner reminiscent of exome capture.
Similar to circular chromosome conformation capture combined with sequencing (4C-seq) before it [32, 33], $\mathrm{CHi}-\mathrm{C}$ parallelizes the one-to-all approach while reducing the cost of standard $\mathrm{Hi}-\mathrm{C}$, and has the potential to map all distal interactions with target regions such as gene promoters. Each capture-based approach also aims to improve upon the resolution of interacting fragments of the genome by applying different DNA cutting enzymes. A similar method, namely chromosome conformation capture coupled with oligonucleotide capture technology (capture-C) [34], wherein genome-wide 3C libraries are fragmented and enriched using oligonucleotide capture technology, was developed to interrogate cis-interactions for 450 promoters. The study reported that promoter interaction probability is highest within $600 \mathrm{~kb}$ up- or downstream of the transcription start site. Similarly, Hi-C capture uses the 4-bp restriction enzyme Mbol for improved resolution, and when applied to generate promoter-anchored interactions for 15,905 promoters revealed 71,984 distal interacting regions in mouse embryonic stem cells (mESCs) [35]. Such techniques may be helpful for validating diseaseassociated variants that modify promoter interactions in mouse models, or to find putative interactions within human syntenic regions. To further overcome resolution limits posed by the usage and availability of restriction sites across the genome, genome-wide chromatin conformation capture with DNase I digestion (DNase Hi-C) was developed [36]. Coupling DNase Hi-C with capture probes for 998 long intervening noncoding RNA (lincRNA) promoters provided approximately 1 -kb resolution of interacting sites in human embryonic stem cells (hESCs) and in the chronic myelogenic leukemia cell line K562. Expansion of this method to all human promoters has the potential to provide the highestresolution maps to date for interacting distal regulatory elements. This is of great importance when considering disease variants that may localize to distal regulatory elements. Target genes are likely regulated by multiple regulatory elements, and fine-mapping the interacting region of the genome that harbors the regulatory element and disease-associated variant is key to determining the likelihood of causality for the variant through dysregulation of gene expression. Below we highlight several examples of how regulatory variant and target gene interactions are being confirmed.

\section{Emerging methodologies for genome architecture and disease}

Understanding how epigenetic modifications and architectural proteins help define chromatin looping is of immense value for advancing our understanding of genome architecture, and specific techniques have been developed to focus on these associated molecular 


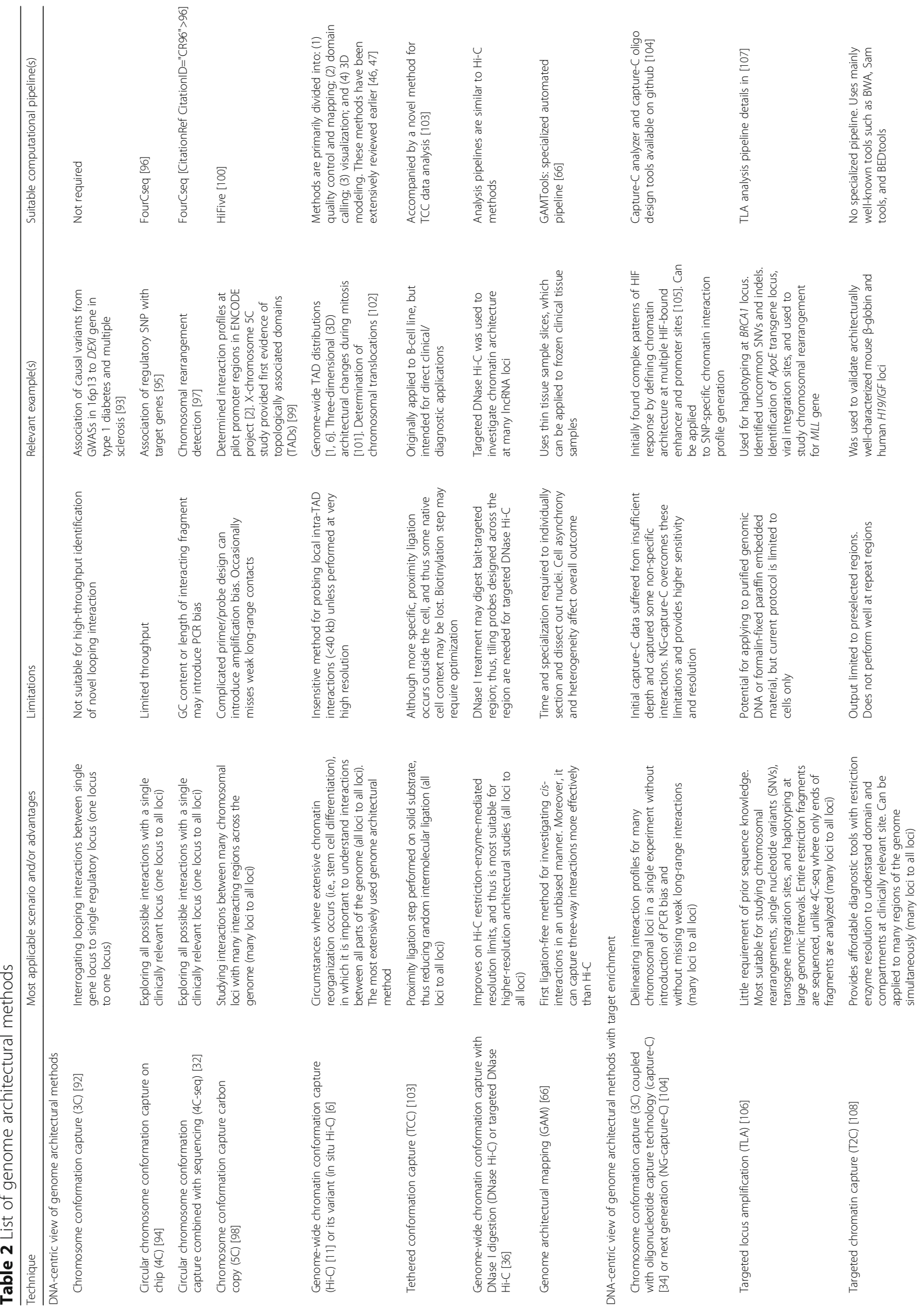




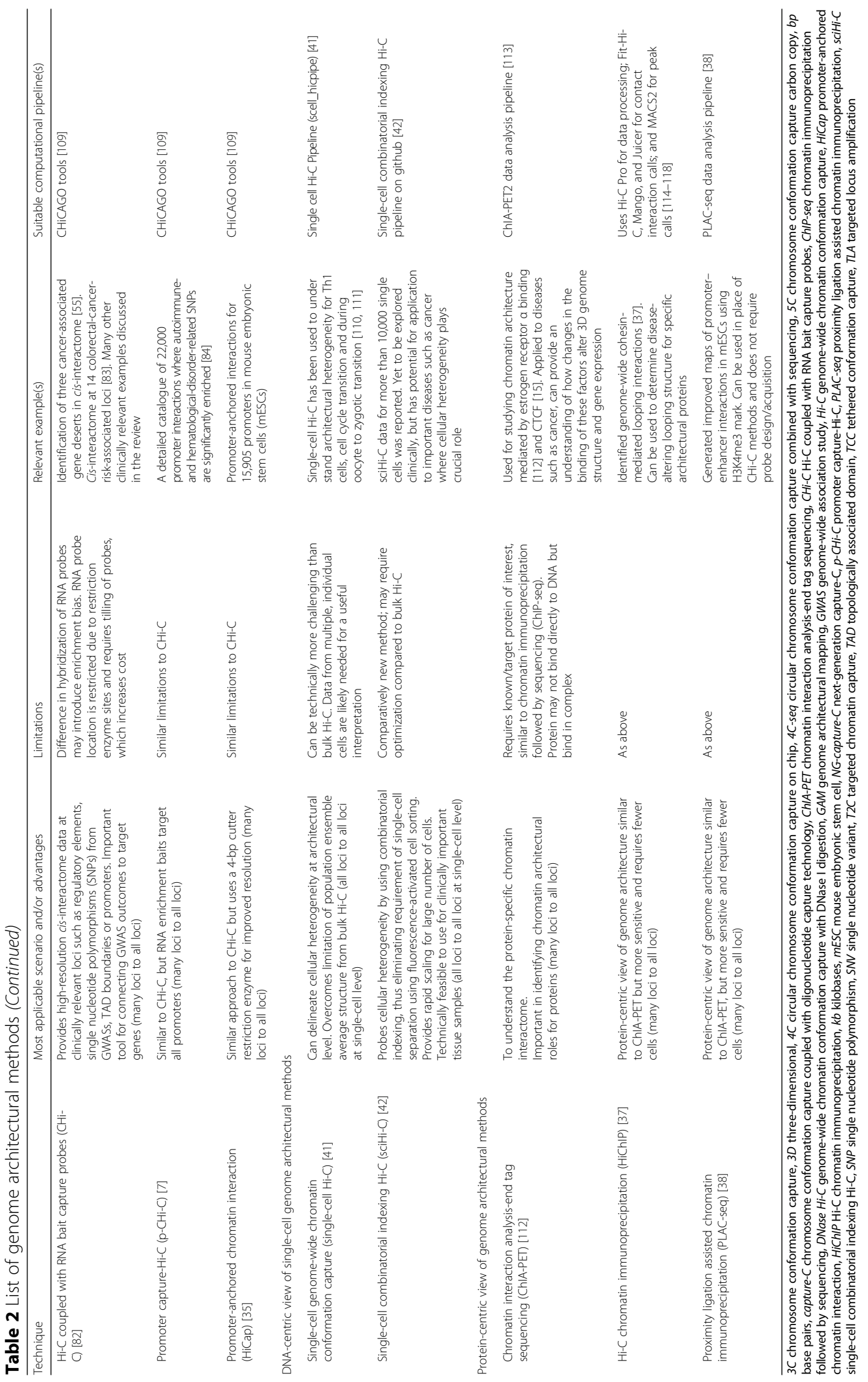


modifiers. For example, HiChIP provides a proteincentric view of genome architecture by coupling ChIP-seq with $\mathrm{Hi}-\mathrm{C}$, and has identified genome-wide cohesin-mediated looping interactions [37]. A similar method, PLAC-seq, targets H3K4me3 histone marks to generate improved maps of promoter-enhancer interactions in mESCs defined by this chromatin modification [38]. Although designed for the same goals as chromatin interaction analysis by paired-end tag sequencing (ChIAPET), HiChIP and PLAC-seq require less starting material, which improves library complexity and signal-to-noise ratios. These methods also work independently of multiple probes, unlike $\mathrm{CHi}-\mathrm{C}$ methods, and thus can be less costly, and ideally prevent probe-binding biases. HiChIP and PLAC-seq should provide useful insights for diseases arising from mutations affecting epigenetic modifiers, TFs, TF-binding loci, and architectural proteins. Below we highlight examples focused on architectural proteins, but future applications could include applying HiChIP or PLAC-seq to numerous chromatin modifiers that are the targets of epigenetic therapies [39], as mutations in several of these modifiers likely alter the 3D genome structure in addition to chromatin structure.

$\mathrm{Hi}-\mathrm{C}$ and $\mathrm{CHi}-\mathrm{C}$ provide information about populationaveraged ensemble structures, as they are performed on millions of cells. (Further pros and cons for capture-based $\mathrm{Hi}-\mathrm{C}$ methods have been reviewed elsewhere [40].) However, there is a growing appreciation for the heterogeneity found among cells in normal as well as diseased tissues, and that such architectural heterogeneity can be revealed at the single-cell level [41]. Combinatorial indexing of $\mathrm{Hi}-\mathrm{C}$ has been developed to distinguish single-cell heterogeneity in 3D architecture more effectively. The addition of combinatorial indexing (Table 1) to Hi-C eliminates the need for cell separation and throughput increases exponentially with each round of indexing [42]. This approach can effectively determine chromosomal inversions, deletions, and rearrangements occurring at the single-cell level within a tumor sample, providing insights into intratumoral evolution with the potential to identify therapeutically relevant drivers or other selective mutations within the lesion.

$\mathrm{Hi}-\mathrm{C}$ catalogues are a valuable resource for understanding disease variants. Integrating $3 \mathrm{D}$ genomic data with genetic data and applying polymer modeling approaches with $\mathrm{Hi}-\mathrm{C}$ catalogues may recapitulate architectural effects of disease variants $[8,43,44]$ and can serve as reference $3 \mathrm{D}$ genome maps for clinically relevant samples-useful for generating testable hypotheses toward therapeutic opportunities. Extensive datasets, protocols, and software for understanding dynamic 3D genome data can be explored at the 4D Nucleome Portal [45], and a range of computational tools are now available for managing and interrogating chromosomal capture datasets, particularly those generated from Hi-C (Table 2) [46-48].

\section{Using genome-editing tools to understand genome architecture in health and disease}

Modern genome-editing applications such as CRISPRCas9 [49] have been employed to study genome architecture and can be broadly classified into three categories: visualization of chromatin dynamics using Cas9 variants; generation of disease models by genome editing; and high-throughput screening for regulatory elements and their effects on chromosomal looping dynamics. Each holds the potential to provide unique insight into disease manifestations.

A limitation to "C"-based techniques is their inability to provide real-time data on chromatin dynamics. Hence, CRISPR-based live cell imaging has been developed to visualize chromatin dynamics by simultaneously tracking multiple genomic loci. CRISPR-based multicolor labeling systems can be used to monitor multiple loci simultaneously. This is achieved through different fluorescently labeled, orthologous catalytically inactive "dead" Cas9 (dCas9) proteins [50], or CRISPRainbow [51], which utilizes engineered single-guide RNA (sgRNAs) such that multiple fluorescent tags can be attached to each guide RNA. Monitoring the localization of these tags can permit tracking of genome-wide topological changes in real time [51] and can be used to validate $\mathrm{Hi}-\mathrm{C}$ data, epigenetic-related architectural changes, and mutation-associated topology changes. Similarly, CASFISH is a FISH variant based on a HaloTag-fused dCas9 that binds fluorescent ligands. Various fluorescent ligands can, therefore, be targeted to different loci in assorted combinations to monitor looping. Although CASFISH has not been applied to live cell imaging, it is technically feasible [52] as another method to track dynamic looping in real time. CRISPR-enabled visualization of real-time architectural changes at selected loci may further define deleterious effects of chromosomal aberrations on architectural arrangement and could have clinical implications in conditions where deletions, duplication, copy number variations, and inversions are causal, as it will provide a means to dissect dysregulation caused by structural variations, and provide a basis for future diagnostic or prognostic developments. These real-time looping visualization techniques can be introduced into induced pluripotent stem cell disease models to pinpoint temporal or molecular stages in which altered regulatory loops or structural elements begin to affect cell function.

CRISPR-Cas9 methodologies can be used for targeted genome editing to reproduce disease-specific mutations in cell lines or model organisms. This has thus far largely 
been achieved through deletions of coding regions. Currently, efforts are underway to delete or modify regulatory elements to understand non-coding disease mutations. Recently, Lupiáñez and colleagues showed that topological chromosomal changes resulting in malformation of human limbs can be elegantly recapitulated in the mouse using CRISPR-Cas9 [53]. CRISPR-Cas9 tools have also been employed to confirm that disruption of architectural boundaries in nonmalignant cells leads to activation of proto-oncogenes [54]. Similarly, genome editing was used to understand the effects of CTCF-binding site (CBS) orientation and their looping pattern by inverting the CBS orientation, which led to altered expression of target genes due to change in looping direction [55]. Considering the versatility of CRISPR-Cas9, a multitude of disease models based on genetic and structural variants are sure to follow in both animal and cellular systems.

Because 3D genome architecture and epigenetic changes are intertwined, targeted modification of epigenetic factors could be instrumental to understanding structural changes induced by such events. A large repertoire of dCas 9 proteins linked with different epigenetic modifiers broadens the applicability of these enzymes to epigenome editing. Using dCas9 fused with either the TET1 or DNMT3A methylation-modifying enzyme demonstrates the impact of DNA methylation on distal enhancer regulation, CTCF-mediated looping, and influencing changes in overall DNA architecture [56]. Similarly, forms of dCas9 facilitating transcriptional activation have been used to modify epigenomic landscapes, which may in turn change the architectural landscape of extremely long-range promoter-promoter interactions [57-59]. High-throughput epigenome editing techniques have been developed, as well as screening methods for phenotypes resulting from epigenetic changes [60-62]. Fulco and colleagues applied genomewide, high-throughput CRISPR interference (CRISPRi) screens to explore novel enhancers surrounding the $M Y C$ and GATA1 loci, which influence proliferative activity in a leukemia model-demonstrating the utility of this technique to explore cis-regulatory influence on disease-relevant phenotypes [63]. Similar epigenomic regulatory element screening can be performed using dCas9-KRAB for repression and dCas9-p300 for activation. For example, a recent study used CRISPR-Cas9based epigenetic regulatory element screening (CERES) to identify novel regulatory elements of the $\beta$-globin and HER2 loci in human cancer cell lines [64]. More recently, the CRISPRi approach was paired with combinatorial barcoding and single-cell RNA-sequencing (RNAseq), termed Mosaic-seq, and demonstrated the importance of defining epistatic interactions between enhancer elements to fully understand their effect on target gene expression [65]. Targeting enhancers in these assays assumes an impact on target genes as measured by RNAseq, which may not be true for all enhancers (i.e., temporarily phenotypic enhancer (Temp) enhancers; see below) [60]. These high-throughput approaches will continue to be developed towards genome-scale interrogation and will further shed light on the capacity in which distal elements drive looping structure. Although CRISPR-Cas9-based genome-editing approaches are promising, they still suffer from off-targeting. To address this, multiple strategies such as ribonucleoprotein (RNP)-based orthologues of Cas9 and modifying sgRNAs are being investigated.

Distal regulatory elements are brought into spatial proximity with their target genes through smaller, likely intra-TAD loops often referred to as regulatory loops. The deletion and repression of distal regulatory elements are expected to influence the architectural landscape. A number of studies discussed below suggest putative complex regulatory three-way interaction-wherein multiple enhancers can regulate a common promoter [43], or multiple promoters converge at a common enhancer [66], or promoter-promoter interactions-wherein enhancer function is attributed to a promoter [67-69]. Such complex regulatory loops are presumably necessary for accurate control of gene expression, and therefore probably differ across cell types or within a disease context, such as overexpression of oncogenes in cancer cells. dCas9 epigenetic modifiers are a valuable novel technology for robust and high-throughput modeling of 3D architectural-based pathologies.

\section{Genome architecture dysregulation and disease pathogenesis}

It is now understood that many disease-associated mutations reside in non-coding regions of the genome; however, primary sequencing has been limited to date for defining precise pathological mechanisms for these nongenic variants. Evidence exists that mutation type and rate are dependent on primary DNA sequence as well as tertiary DNA arrangement (for review, see [70]). It is notable that mutation rates across the genome vary [71], and that chromatin architecture can be highly variable through different developmental stages and between cell types. As a result, the fluctuating mutation rate is strongly related to changes in DNA accessibility [72], and it was recently reported that the mutational contours of cancer are largely determined by the chromatin landscape of the cell type of origin [73]. It is well established that regulatory elements overlap with DNase I hypersensitive sites (DHSs). DHSs are known to be under purifying selection [74]. Interestingly, the mutation rate within DHSs also varies between cell states and types; that is, pluripotent cells and immortalized cells show higher mutation rates in DHSs when compared with the DHSs of differentiated cells [74]. This observed 
heterogeneous mutational spectrum across cell types aligns well with the cell-type specificity of intra-TAD interactions. Thus, it is feasible that differences in mutation rate may provide a dynamic adaptive mutation range to regulatory elements for fitting in different regulatory circuits. Moreover, mutations are the basis of disease, and this interrelation with chromatin states points toward the importance of 3D genome architecture for a detailed understanding of pathogenesis. For example, phenotypes including limb malformations and proto-oncogene activation have been observed arising from detrimental mutations that disrupt existing TAD boundaries or create spurious new TAD interactions [53, 54] (Table 3).

Disruption of factors regulating genome architecture can cause deleterious changes in genome topology. For example, deletions, duplications, or changes in the epigenetic landscape that lead to aberrant binding of CTCF or associated architectural proteins and lncRNAs in turn alter TAD structure. The master regulator of DNA architecture, CTCF, has been implicated in a multitude of diseases. Targeted therapies related to these disruptions are still lacking but are of high clinical interest for cases in which hypermethylation in cancer cells disrupts CTCF binding, with available demethylating agents having the potential to restore CTCF binding (see Table 3 for representative examples).

\section{Generation of 3D genome catalogues and integrative analysis}

As the majority of significant non-coding variants from GWASs fall within DNase hypersensitive regions such as enhancers, silencers, or insulators [75, 76], determining how distal, non-coding regulatory variants impact gene expression and in turn have pathological consequences is important. High-resolution interaction maps will prove essential in this effort and have already revealed novel insights into the complexity of disease genetics and cis-regulation. Here, we highlight several recent studies.

Recent 3D architectural studies in the brain have emphasized their potential for elucidating complex mechanisms of neuropsychiatric disorders that are not fully understood (for review see [77, 78]). In brain function, long-term potentiation (LTP) and synaptogenesis are very dynamic events that need to be regulated by rapid gene expression changes. Therefore, when the impulse for LTP or synaptogenesis is present, rapid dynamic looping may load transcriptional-machinery-rich enhancers to the promoter for rapid gene regulation. These kinds of neuronal-impulse-induced architectural movements were observed previously for the brainderived neurotrophic factor $(B d n f)$ locus in mice and satellite DNA loci $[79,80]$. Detailed Hi-C maps for cortical and germinal brain regions identified increased

Table 3 Architectural changes and disease

\begin{tabular}{|c|c|c|c|}
\hline Architectural component & Disease phenotype or mutation effect & Underlying cause or architectural change & References \\
\hline CTCF & Silencing of tumor suppressor XAF1 & $\begin{array}{l}\text { Hypermethylation of CTCF-binding site near } \\
\text { XAF1 promoter }\end{array}$ & [119] \\
\hline CTCF & $\begin{array}{l}\text { Illegitimate enhancer access of PDGFRA } \\
\text { and its overexpression }\end{array}$ & $\begin{array}{l}\text { Hypermethylation of CTCF-binding site due to } \\
\text { IDH mutation and disruption of TAD boundary }\end{array}$ & [120] \\
\hline CTCF & Human limb malformation & $\begin{array}{l}\text { Altered TAD structure surrounding WNT6/IHH/ } \\
\text { EPHA4/PAX3 due to deletion, duplication or } \\
\text { inversion in CTCF boundary element }\end{array}$ & [53] \\
\hline CTCF-cohesin & $\begin{array}{l}\text { Activation of proto-oncogenes in T-cell } \\
\text { acute lymphoblastic leukemia }\end{array}$ & $\begin{array}{l}\text { Microdeletion of insulated boundary and } \\
\text { aberrant access of enhancer to oncogene }\end{array}$ & [54] \\
\hline $\begin{array}{l}\text { Cohesin loading factor NIPBL } \\
\text { in } 50 \% \text { of cases }\end{array}$ & Cornelia de Lange syndrome & $\begin{array}{l}\text { NIPBL mutation leads to chromatin decompaction } \\
\text { in gene-rich regions. Chromatin architectural } \\
\text { dysregulation suspected, but no direct evidence }\end{array}$ & {$[19,121]$} \\
\hline MED12 & $\begin{array}{l}\text { X-linked mental retardation Opitz } \\
\text { Kaveggia syndrome }\end{array}$ & $\begin{array}{l}\text { Recurrent mutation R961W in MED12, which } \\
\text { affects its interaction with ncRNA a-1 and ncRNA } \\
\text { a-3, and, therefore, likely disruption of regulatory } \\
\text { loops mediated by MED12 and ncRNAs }\end{array}$ & {$[122,123]$} \\
\hline Lamin A & Hutchinson-Gilford Progeria syndrome & $\begin{array}{l}\text { Point mutation in lamin A, loss of } \mathrm{H} 3 \mathrm{~K} 27 \mathrm{me} 3 \text {, } \\
\text { which in turn leads to global loss of spatial } \\
\text { chromatin structure at the nuclear lamina }\end{array}$ & [124-126] \\
\hline $\begin{array}{l}\text { Long non-coding RNA (InCRNA) } \\
\text { CCAT1-L }\end{array}$ & Colorectal cancer & $\begin{array}{l}\text { This IncRNA is transcribed from an } 8 \mathrm{q} 24 \text { gene } \\
\text { desert and interacts with CTCF to form looping structures } \\
\text { at the MYC locus, leading to overexpression }\end{array}$ & {$[127]$} \\
\hline InCRNA CISR-ACT & Brachydactyly type $\mathrm{E}$ & $\begin{array}{l}\text { Translocation-mediated disruption of cis-interactions } \\
\text { between a IncRNA and the parathyroid hormone-like } \\
\text { hormone (PTHLH) gene, reducing its expression level }\end{array}$ & [128] \\
\hline
\end{tabular}


promoter-enhancer interactions compared with other tissues [81]. The authors found that novel human-gained enhancers showed significant overlap with lineagespecific lncRNAs and 108 significant schizophreniaassociated variants. This study and others like it have important implications for disorders and diseases outside the nervous system.

The influence of modifications to distal regulatory elements spans tissue types as well as disease types. An extensive study of 21 different cell and tissue types determined thousands of frequently interacting enhancer regions (FIREs) using Hi-C maps [43]. These FIREs are tissue specific in nature and most correspond to active enhancers, as defined by chromatin state. Among all the FIREs detected, 354 are classified as super-enhancers, 2800 as typical enhancers, and 1615 as new or putative enhancers that were not previously known. FIREs for 456 disease-associated single nucleotide polymorphisms (SNPs) and quantitative trait loci (QTLs) were also identified. Distinct disease-associated FIREs were found in specific tissues or cell types, which further strengthens the association; for example, Alzheimer's SNPs were found in brain-specific FIREs, and SNPs for acute lymphoblastic leukemia were found in GM12878specific super-FIREs. The tissue and cell specificity of these interaction regions may help reveal how disease variants manifest in tissue-specific phenotypes.

$\mathrm{CHi}-\mathrm{C}$ methods can substantially aid in connecting disease-associated SNPs with target genes, and have already advanced our understanding of the genetic basis for many diseases. $\mathrm{CHi}-\mathrm{C}$ was first utilized to understand the interactions of three cancer risk-associated regions that lie in gene deserts. In this approach, 519 bait probes were used to capture regions interacting with these gene desert loci and identified interacting regions that included protein-coding genes, lncRNAs, and cancer-associated SNPs [82]. Similarly, CHi-C was also employed in defining interactomes for 14 colorectalcancer-risk-associated loci [83]. These distal interacting regions of disease risk likely harbor regulatory elements that are altered and confer disease; therefore, this has prognostic potential by identifying causal variants.

A detailed catalogue of 22,000 promoter interactions was generated using promoter $\mathrm{CHi}-\mathrm{C}$ for two blood cell types: the lymphoblast line GM12878 and CD34. hematopoietic progenitor cells. 3D interaction data indicated that SNPs associated with autoimmune and other hematological disorders were significantly enriched at interacting distal regulatory sites of targeted promoters [84], suggesting that these genes are likely dysregulated in the disease state. Similarly, $\mathrm{CHi}-\mathrm{C}$ was also applied to study autoimmune-disease associated SNPs in GM12878 B-cell and Jurkat T-cell lines. These findings demonstrated that different autoimmune-associated variants interact with common gene promoters, which are presumably dysregulated. They also contradict the longheld assumption that disease-causing genes and their associated variants should be in close linkage disequilibrium (LD) to impart an effect [85]. Another recent study leveraged existing $\mathrm{Hi}-\mathrm{C}$ data to determine that variants at regulatory elements outside of LD blocks interacted with genes or their enhancers harboring linked SNPs to impact gene expression and disease risk [86]. These variants were termed "outside variants" based on their location outside of LD blocks.

Another study generated extensive catalogues of distal genomic regions that interact with promoters, or promoter-interacting regions (PIRs), in 17 primary hematopoietic cell types [7]. The authors linked 2500 novel SNPs to putative disease-associated genes related to blood and autoimmune disorders. It was observed that PIRs were highly cell type specific, and, as noted above, this implicates which variants are likely drivers of cell-specific phenotypes due to their location in either cell-specific enhancers or regulatory loops. Novel putative enhancers, which lacked typical enhancer chromatin signatures such as histone methylation, were identified through these captured interactions and showed an additive effect on gene expression. This additive effect indicates that more than one enhancer interacts on a particular target. This one-target-multi-enhancer model suggests the evolution of fail-safe transcriptional circuits, wherein mutation in one or two enhancers may not lead to the breakdown of target gene activation [7]. In support of this model, Temp enhancers were recently described in hESCs surrounding the POU5F1 locus, which encodes OCT4, a master regulator of ESCs [60]. CRISPR-Cas9-targeted deletion of certain enhancers led to only a temporary reduction of the OCT4-encoding transcript, which eventually returned to normal levels. This could have important implications in understanding how epistatic relationships between cis-regulatory elements are used to maintain cellular homeostasis.

A central goal of modern genomics research is to translate GWAS discoveries into therapeutic outcomes. A CHi-C study of a critical autoimmune risk locus on chromosome 6q23 reported that different autoimmune related disorders such as rheumatoid arthritis, psoriasis, and type 1 diabetes were regulated by a common intergenic enhancer, suggesting a "transcription factory"-like structure. Moreover, the research identified the involvement of a novel gene, IL20RA, and suggested that treatment using monoclonal antibodies targeting its ligand IL20 may provide better therapeutic outcome for the related autoimmune disorders [87]. Importantly, autoimmune diseases, and similarly neurological disorders, often share disease-associated variants; thus, future investigation of looping structures could reveal common 
mechanisms for multiple diseases within these broader categories.

Finally, Hi-C can be used for genome assembly [88], which has important implications for the study of disease. For example, genome assembly is proving important in determining copy number variants and translocation in cancer, and may also have applications to neurological disorders and others in which chromosomal deletions, inversions, or duplications are drivers of the disease. It is being used to phase genomes for haplotype structures [89], which will aid our understanding of inherited alleles and their variants, relevant for rare inherited diseases. $\mathrm{Hi}-\mathrm{C}$ is also being used for rapid genome assembly of pathogens [90], as the proximity ligation, on which the method relies, enables assembly without prior knowledge of genome sequence or structure. This is sure to influence how we understand hostpathogen interactions and treatments.

\section{Conclusions and future perspectives}

The applications and development of technologies to investigate $3 \mathrm{D}$ genome architecture are rapidly changing how we view genomics. Advances in our understanding of architectural arrangements for precise loci using $\mathrm{Hi}-\mathrm{C}$, $\mathrm{CHi}-\mathrm{C}$, and allied techniques are helping to associate noncoding (regulatory) disease variants (SNPs) with the most probable target genes, and could explain pathomechanisms mediated via distal regulatory variants. Moreover, the identification of genes interacting with disease-associated regulatory variants provides a basis for determining enriched signaling pathways involved in the pathogenesis of diseases, which may lead to therapeutic interventions that are more readily targetable than those aimed at the variant or TF that binds the site. The recent application of CRISPR-based tools and integrated "C"-based techniques are likely to further our understanding of the relationship between regulation and 3D architecture.

Multiple genetic disorders, as discussed above, have been associated with altered architectural modules. Combining $\mathrm{Hi}-\mathrm{C}$ data with genome-editing tools may lead to therapeutic outcomes via cell-based therapy and the introduction or removal of architectural modules. Similarly, site-specific epigenome editing has also paved the way for the investigation of directed architectural changes. Catalogues of interaction maps from various cells and tissues now serve as references for comparing future 3D genome maps from diseased states. Computational tools to facilitate analysis of these new datasets are concurrently being developed. Collectively, this will further the clinical applications of 3D genomics.

Increasing evidence indicates the strong possibility of transcription factory or hub-like structures in cells, in which multiple enhancers, silencers or other elements may regulate one or more promoters together [7, 66, 91].
Screening the complex epistatic interactions within these regulatory loops may uncover novel mechanisms of disease resulting from disrupted architectural regulation. In summary, we are beginning to learn if or how singlenucleotide and structural variants impact genome folding. The rapid technological advances in this field have highlighted the importance of studying 3D genomics to improve prognostic, diagnostic, and potentially therapeutic outcomes.

\section{Abbreviations}

3C: Chromosome conformation capture; 3D: Three-dimensional; 4C: Circular chromosome conformation capture on chip; 4C-seq: Circular chromosome conformation capture on chip combined with sequencing; 5C: Chromosome conformation capture carbon copy; bp: Base pairs; capture-C: Chromosome conformation capture coupled with oligonucleotide capture technology; CBS: CTCF-binding site; CERES: CRISPR-Cas9-based epigenetic regulatory element screening; ChIA-PET: Chromatin interaction analysis-end tag sequencing; CHi-C: Hi-C coupled with RNA bait capture probes; ChIP-seq: chromatin immunoprecipitation followed by sequencing; CRISPR: Clustered regularly interspaced short palindromic repeats; CRISPRi: CRISPR interference; DHS: DNase I hypersensitive site; DNase Hi-C: Genome-wide chromatin conformation capture with DNase I digestion; FIREs: Frequently interacting enhancer regions; FISH: Fluorescence in situ hybridization; GAM: Genome architectural mapping; GWAS: Genome-wide association study; hESC: Human embryonic stem cell; Hi$\mathrm{C}$ : Genome-wide chromatin conformation capture; Hi-Cap: Hi-C capture;

HiChIP: Hi-C chromatin immunoprecipitation; kb: Kilobase; LAD: Lamina-associated domain; LD: Linkage disequilibrium; lincRNA: Long intergenic non-coding RNA; IncRNA: Long non-coding RNA; LTP: Long-term potentiation; Mb: Megabase; mESC: Mouse embryonic stem cell; NG-capture-C: Next-generation capture-C; PIR: Promoter-interacting regions; PLAC-seq: Proximity ligation assisted chromatin immunoprecipitation; QTL: Quantitative trait loci; RNA-seq: RNA sequencing; sciHiC: Single-cell combinatorial indexing Hi-C; sgRNA: Single guide RNA; SNP: Single nucleotide polymorphism; SNV: Single nucleotide variant; T2C: Targeted chromatin capture; TAD: Topologically associated domain; TCC: Tethered conformation capture; Temp: Temporarily phenotypic; TF: Transcription factor; TLA: Targeted locus amplification

\section{Acknowledgements}

We would like to thank Dr. Rini Shah for critical comments on the manuscript. We apologize to those whose work was not cited due to space limitations.

\section{Funding}

RDH is supported by the National Institutes of Health: NIAMS and NIDDK; and the US-Israel Binational Science Foundation.

\section{Authors' contributions}

AM and RDH wrote and edited the manuscript. Both authors read and approved the final manuscript.

\section{Competing interests}

The authors declare that they have no competing interests.

\section{Publisher's Note}

Springer Nature remains neutral with regard to jurisdictional claims in published maps and institutional affiliations.

Published online: 30 September 2017

\section{References}

1. Dixon JR, Selvaraj S, Yue F, Kim A, Li Y, Shen Y, et al. Topological domains in mammalian genomes identified by analysis of chromatin interactions. Nature. 2012;485:376-80.

2. Nora EP, Lajoie BR, Schulz EG, Giorgetti L, Okamoto I, Servant N, et al. Spatial partitioning of the regulatory landscape of the X-inactivation centre. Nature. 2012:485:381-5.

3. Hnisz D, Day DS, Young RA. Insulated neighborhoods: structural and functional units of mammalian gene control. Cell. 2016;167:1188-200. 
4. Splinter E, Heath $H$, Kooren J, Palstra R-J, Klous P, Grosveld F, et al. CTCF mediates long-range chromatin looping and local histone modification in the $\beta$-globin locus. Genes Dev. 2006;20:2349-54.

5. Hadjur S, Williams LM, Ryan NK, Cobb BS, Sexton T, Fraser P, et al. Cohesins form chromosomal cis-interactions at the developmentally regulated IFNG locus. Nature. 2009:460:410-3.

6. Rao SSP, Huntley MH, Durand NC, Stamenova EK, Bochkov ID, Robinson JT, et al. A 3D map of the human genome at kilobase resolution reveals principles of chromatin looping. Cell. 2015;162:687-8

7. Javierre BM, Burren OS, Wilder SP, Kreuzhuber R, Hill SM, Sewitz $S$, et al. Lineage-specific genome architecture links enhancers and non-coding disease variants to target gene promoters. Cell. 2016;167:1369-84.

8. Freire-Pritchett P, Schoenfelder S, Várnai C, Wingett SW, Cairns J, Collier AJ, et al. Global reorganisation of cis-regulatory units upon lineage commitment of human embryonic stem cells. Elife. 2017;6. doi:10.7554/ eLife.21926

9. Zhan Y, Mariani L, Barozzi I, Schulz EG, Blüthgen N, Stadler M, et al. Reciprocal insulation analysis of Hi-C data shows that TADs represent a functionally but not structurally privileged scale in the hierarchical folding of chromosomes. Genome Res. 2017;27:479-90.

10. Fraser J, Ferrai C, Chiariello AM, Schueler M, Rito T, Laudanno G, et al. Hierarchical folding and reorganization of chromosomes are linked to transcriptional changes in cellular differentiation. Mol Syst Biol. 2015;11:852.

11. Lieberman-Aiden E, van Berkum NL, Williams L, Imakaev M, Ragoczy T, Telling $A$, et al. Comprehensive mapping of long-range interactions reveals folding principles of the human genome. Science. 2009;326:289-93.

12. Meaburn KJ, Misteli T. Cell biology: chromosome territories. Nature. 2007; 445:379-781.

13. Bonev B, Cavalli G. Organization and function of the $3 D$ genome. Nat Rev Genet. 2016;17:661-78.

14. Ong C-T, Corces VG. CTCF: an architectural protein bridging genome topology and function. Nat Rev Genet. 2014;15:234-46.

15. Handoko L, Xu H, Li G, Ngan CY, Chew E, Schnapp M, et al. CTCF-mediated functional chromatin interactome in pluripotent cells. Nat Genet. 2011;43:630-8.

16. Bell AC, Felsenfeld G. Methylation of a CTCF-dependent boundary controls imprinted expression of the lgf2 gene. Nature. 2000;405:482-5.

17. Hark AT, Schoenherr CJ, Katz DJ, Ingram RS, Levorse JM, Tilghman SM. CTCF mediates methylation-sensitive enhancer-blocking activity at the H19/lgf2 locus. Nature. 2000;405:486-9.

18. Nasmyth $\mathrm{K}$, Haering $\mathrm{CH}$. Cohesin: its roles and mechanisms. Annu Rev Genet. 2009;43:525-58.

19. Kagey MH, Newman JJ, Bilodeau S, Zhan Y, Orlando DA, van Berkum NL, et al. Mediator and cohesin connect gene expression and chromatin architecture. Nature. 2010;467:430-5.

20. Zuin J, Dixon JR, van der Reijden MIJA, Ye Z, Kolovos P, Brouwer RWW, et al. Cohesin and CTCF differentially affect chromatin architecture and gene expression in human cells. Proc Natl Acad Sci U S A. 2014;111:996-1001.

21. Guelen L, Pagie L, Brasset E, Meuleman W, Faza MB, Talhout W, et al. Domain organization of human chromosomes revealed by mapping of nuclear lamina interactions. Nature. 2008;453:948-51.

22. Engreitz JM, Ollikainen N, Guttman M. Long non-coding RNAs: spatial amplifiers that control nuclear structure and gene expression. Nat Rev Mol Cell Biol. 2016;17:756-70.

23. Krijger PHL, de Laat W. Regulation of disease-associated gene expression in the 3D genome. Nat Rev Mol Cell Biol. 2016;17:771-82.

24. Lupiáñez DG, Spielmann M, Mundlos S. Breaking TADs: how alterations of chromatin domains result in disease. Trends Genet. 2016:32:225-37.

25. Song F, Chen P, Sun D, Wang M, Dong L, Liang D, et al. Cryo-EM study of the chromatin fiber reveals a double helix twisted by tetranucleosomal units. Science. 2014;344:376-80.

26. Ramani V, Shendure J, Duan Z. Understanding spatial genome organization: methods and insights. Genomics Proteomics Bioinformatics. 2016;14:7-20.

27. Sajan SA, Hawkins RD. Methods for identifying higher-order chromatin structure. Annu Rev Genomics Hum Genet. 2012;13:59-82.

28. Schmitt AD, Hu M, Ren B. Genome-wide mapping and analysis of chromosome architecture. Nat Rev Mol Cell Biol. 2016;17:743-55.

29. Barutcu AR, Fritz AJ, Zaidi SK, van Wijnen AJ, Lian JB, Stein JL, et al. C-ing the genome: a compendium of chromosome conformation capture methods to study higher-order chromatin organization. J Cell Physiol. 2016;231:31-5.
30. Davies JOJ, Marieke Oudelaar A, Higgs DR, Hughes JR. How best to identify chromosomal interactions: a comparison of approaches. Nat Methods. 2017;14:125-34.

31. Sati S, Cavalli G. Chromosome conformation capture technologies and their impact in understanding genome function. Chromosoma. 2017;126:33-44.

32. Splinter $E$, de Wit $E$, van de Werken HJG, Klous P, de Laat W. Determining long-range chromatin interactions for selected genomic sites using 4C-seq technology: from fixation to computation. Methods. 2012:58:221-30.

33. Van De Werken HJG, Landan G, Holwerda SJB, Hoichman M, Klous P, Chachik $R$, et al. Robust 4C-seq data analysis to screen for regulatory DNA interactions. Nat Methods. 2012;9:969.

34. Hughes JR, Roberts N, McGowan S, Hay D, Giannoulatou E, Lynch M, et al. Analysis of hundreds of cis-regulatory landscapes at high resolution in a single, high-throughput experiment. Nat Genet. 2014;46:205-12.

35. Sahlén P, Abdullayev I, Ramsköld D, Matskova L, Rilakovic N, Lötstedt B, et al. Genome-wide mapping of promoter-anchored interactions with close to single-enhancer resolution. Genome Biol. 2015;16:156.

36. Ma W, Ay F, Lee C, Gulsoy G, Deng X, Cook S, et al. Fine-scale chromatin interaction maps reveal the cis-regulatory landscape of human lincRNA genes. Nat Methods. 2015;12:71-8.

37. Mumbach MR, Rubin AJ, Flynn RA, Dai C, Khavari PA, Greenleaf WJ, et al. HiChIP: efficient and sensitive analysis of protein-directed genome architecture. Nat Methods. 2016;13:919-22.

38. Fang $R$, Yu M, Li G, Chee S, Liu T, Schmitt AD, et al. Mapping of long-range chromatin interactions by proximity ligation-assisted ChIP-seq. Cell Res. 2016;26:1345-8.

39. Jones PA, Issa J-PJ, Baylin S. Targeting the cancer epigenome for therapy. Nat Rev Genet. 2016;17:630-41.

40. Osborne CS, Mifsud B. Capturing genomic relationships that matter. Chromosome Res. 2017;25:15-24.

41. Nagano T, Lubling Y, Stevens TJ, Schoenfelder S, Yaffe E, Dean W, et al. Single-cell $\mathrm{Hi}-\mathrm{C}$ reveals cell-to-cell variability in chromosome structure. Nature. 2013;502:59-64.

42. Ramani V, Deng X, Qiu R, Gunderson KL, Steemers FJ, Disteche CM, et al. Massively multiplex single-cell Hi-C. Nat Methods. 2017;14:263-6.

43. Schmitt AD, Hu M, Jung I, Xu Z, Qiu Y, Tan CL, et al. A compendium of chromatin contact maps reveals spatially active regions in the human genome. Cell Rep. 2016;17:2042-59.

44. Giorgetti L, Galupa R, Nora EP, Piolot T, Lam F, Dekker J, et al. Predictive polymer modeling reveals coupled fluctuations in chromosome conformation and transcription. Cell. 2014;157:950-63.

45. 4D Nucleome Portal. http://www.4dnucleome.org

46. Ay F, Noble WS. Analysis methods for studying the 3D architecture of the genome. Genome Biol. 2015;16:183.

47. Forcato M, Nicoletti C, Pal K, Livi CM, Ferrari F, Bicciato S. Comparison of computational methods for Hi-C data analysis. Nat Methods. 2017;14:679-85.

48. Yardımcı GG, Noble WS. Software tools for visualizing Hi-C data. Genome Biol. 2017;18:26.

49. Jinek M, Chylinski K, Fonfara I, Hauer M, Doudna JA, Charpentier E. A programmable dual-RNA-duided DNA endonuclease in adaptive bacterial immunity. Science. 2012;337:816-21.

50. Ma H, Naseri A, Reyes-Gutierrez P, Wolfe SA, Zhang S, Pederson T. Multicolor CRISPR labeling of chromosomal loci in human cells. Proc Natl Acad Sci U S A. 2015:112:3002-7.

51. Ma H, Tu L-C, Naseri A, Huisman M, Zhang S, Grunwald D, et al. Multiplexed labeling of genomic loci with dCas9 and engineered sgRNAs using CRISPRainbow. Nat Biotechnol. 2016;34:528-30.

52. Deng W, Shi $X$, Tjian R, Lionnet T, Singer RH. CASFISH: CRISPR/Cas9-mediated in situ labeling of genomic loci in fixed cells. Proc Natl Acad Sci U S A. 2015;112:11870-5.

53. Lupiáñez DG, Kraft K, Heinrich V, Krawitz P, Brancati F, Klopocki E, et al. Disruptions of topological chromatin domains cause pathogenic rewiring of gene-enhancer interactions. Cell. 2015;161:1012-25.

54. Hnisz D, Weintraub AS, Day DS, Valton A-L, Bak RO, Li CH, et al. Activation of proto-oncogenes by disruption of chromosome neighborhoods. Science. 2016:351:1454-8.

55. Guo Y, Xu Q, Canzio D, Shou J, Li J, Gorkin DU, et al. CRISPR inversion of CTCF sites alters genome topology and enhancer/promoter function. Cell. 2015;162:900-10.

56. Liu XS, Wu H, Ji X, Stelzer $Y$, Wu X, Czauderna S, et al. Editing DNA methylation in the mammalian genome. Cell. 2016;167:233-47. 
57. Konermann S, Brigham MD, Trevino AE, Joung J, Abudayyeh $\bigcirc O$, Barcena C, et al. Genome-scale transcriptional activation by an engineered CRISPR-Cas9 complex. Nature. 2015;517:583-8.

58. Cheng AW, Wang H, Yang H, Shi L, Katz Y, Theunissen TW, et al. Multiplexed activation of endogenous genes by CRISPR-on, an RNA-guided transcriptional activator system. Cell Res. 2013;23:1163-71.

59. Joshi O, Wang S-Y, Kuznetsova T, Atlasi Y, Peng T, Fabre PJ, et al. Dynamic reorganization of extremely long-range promoter-promoter interactions between two states of pluripotency. Cell Stem Cell. 2015;17:748-57.

60. Diao Y, Li B, Meng Z, Jung I, Lee AY, Dixon J, et al. A new class of temporarily phenotypic enhancers identified by CRISPR/Cas9-mediated genetic screening. Genome Res. 2016;26:397-405.

61. Rajagopal N, Srinivasan S, Kooshesh K, Guo Y, Edwards MD, Banerjee B, et al. High-throughput mapping of regulatory DNA. Nat Biotechnol. 2016;34:167-74

62. Sanjana NE, Wright J, Zheng K, Shalem O, Fontanillas P, Joung J, et al. Highresolution interrogation of functional elements in the noncoding genome. Science. 2016;353:1545-9.

63. Fulco CP, Munschauer M, Anyoha R, Munson G, Grossman SR, Perez EM, et al. Systematic mapping of functional enhancer-promoter connections with CRISPR interference. Science. 2016;354:769-73.

64. Klann TS, Black JB, Chellappan M, Safi A, Song L, Hilton IB, et al. CRISPR-Cas9 epigenome editing enables high-throughput screening for functional regulatory elements in the human genome. Nat Biotechnol. 2017;35:561-8.

65. Xie S, Duan J, Li B, Zhou P, Hon GC. Multiplexed engineering and analysis of combinatorial enhancer activity in single cells. Mol Cell. 2017;66:285-99.

66. Beagrie RA, Scialdone A, Schueler M, Kraemer DCA, Chotalia M, Xie SQ, et al. Complex multi-enhancer contacts captured by genome architecture mapping. Nature. 2017;543:519-24.

67. Dao LTM, Galindo-Albarrán AO, Castro-Mondragon JA, Andrieu-Soler C, Medina-Rivera A, Souaid C, et al. Genome-wide characterization of mammalian promoters with distal enhancer functions. Nat Genet. 2017:49:1073-81.

68. Diao Y, Fang R, Li B, Meng Z, Yu J, Qiu Y, et al. A tiling-deletion-based genetic screen for cis-regulatory element identification in mammalian cells. Nat Methods. 2017;14:629-35.

69. Catarino RR, Neumayr C, Stark A. Promoting transcription over long distances. Nat Genet. 2017;49:972-3.

70. Makova KD, Hardison RC. The effects of chromatin organization on variation in mutation rates in the genome. Nat Rev Genet. 2015;16:213-23.

71. Wolfe KH, Sharp PM, Li WH. Mutation rates differ among regions of the mammalian genome. Nature. 1989;337:283-5.

72. Schuster-Böckler B, Lehner B. Chromatin organization is a major influence on regional mutation rates in human cancer cells. Nature. 2012;488:504-7.

73. Polak P, Karlić R, Koren A, Thurman R, Sandstrom R, Lawrence MS, et al. Cellof-origin chromatin organization shapes the mutational landscape of cancer. Nature. 2015;518:360-4.

74. Thurman RE, Rynes E, Humbert R, Vierstra J, Maurano MT, Haugen E, et al. The accessible chromatin landscape of the human genome. Nature. 2012:489:75-82.

75. Maurano MT, Humbert R, Rynes E, Thurman RE, Haugen E, Wang H, et al. Systematic localization of common disease-associated variation in regulatory DNA. Science. 2012;337:1190-5.

76. Schaub MA, Boyle AP, Kundaje A, Batzoglou S, Snyder M. Linking disease associations with regulatory information in the human genome. Genome Res. 2012:22:1748-59.

77. Medrano-Fernández A, Barco A. Nuclear organization and 3D chromatin architecture in cognition and neuropsychiatric disorders. Mol Brain. 2016;9:83.

78. Rajarajan P, Gil SE, Brennand KJ, Akbarian S. Spatial genome organization and cognition. Nat Rev Neurosci. 2016;17:681-91.

79. Billia F, Baskys A, Carlen PL, De Boni U. Rearrangement of centromeric satellite DNA in hippocampal neurons exhibiting long-term potentiation. Brain Res Mol Brain Res. 1992;14:101-8.

80. Walczak A, Szczepankiewicz AA, Ruszczycki B, Magalska A, Zamlynska K, Dzwonek J, et al. Novel higher-order epigenetic regulation of the Bdnf gene upon seizures. J Neurosci. 2013;33:2507-11.

81. Won H, de la Torre-Ubieta L, Stein JL, Parikshak NN, Huang J, Opland CK, et al. Chromosome conformation elucidates regulatory relationships in developing human brain. Nature. 2016;538:523-7.

82. Dryden NH, Broome LR, Dudbridge F, Johnson N, Orr N, Schoenfelder S, et al. Unbiased analysis of potential targets of breast cancer susceptibility loci by Capture Hi-C. Genome Res. 2014;24:1854-68.
83. Jäger R, Migliorini G, Henrion M, Kandaswamy R, Speedy HE, Heindl A, et al. Capture Hi-C identifies the chromatin interactome of colorectal cancer risk loci. Nat Commun. 2015;6:6178.

84. Mifsud B, Tavares-Cadete F, Young AN, Sugar R, Schoenfelder S, Ferreira L, et al. Mapping long-range promoter contacts in human cells with highresolution capture Hi-C. Nat Genet. 2015;47:598-606.

85. Martin P, McGovern A, Orozco G, Duffus K, Yarwood A, Schoenfelder S, et al. Capture $\mathrm{Hi}-\mathrm{C}$ reveals novel candidate genes and complex long-range interactions with related autoimmune risk loci. Nat Commun. 2015;6:10069.

86. Corradin O, Cohen AJ, Luppino JM, Bayles IM, Schumacher FR, Scacheri PC. Modeling disease risk through analysis of physical interactions between genetic variants within chromatin regulatory circuitry. Nat Genet. 2016;48:1313-20.

87. McGovern A, Schoenfelder S, Martin P, Massey J, Duffus K, Plant D, et al. Capture Hi-C identifies a novel causal gene, IL20RA, in the pan-autoimmune genetic susceptibility region 6q23. Genome Biol. 2016;17:212.

88. Burton JN, Adey A, Patwardhan RP, Qiu R, Kitzman JO, Shendure J. Chromosome-scale scaffolding of de novo genome assemblies based on chromatin interactions. Nat Biotechnol. 2013;31:1119-25.

89. Selvaraj S, R Dixon J, Bansal V, Ren B. Whole-genome haplotype reconstruction using proximity-ligation and shotgun sequencing. Nat Biotechnol. 2013:31:1111-8.

90. Dudchenko O, Batra SS, Omer AD, Nyquist SK, Hoeger M, Durand NC, et al. De novo assembly of the Aedes aegypti genome using $\mathrm{Hi}-\mathrm{C}$ yields chromosome-length scaffolds. Science. 2017;356:92-5.

91. Stevens TJ, Lando D, Basu S, Atkinson LP, Cao Y, Lee SF, et al. 3D structures of individual mammalian genomes studied by single-cell Hi-C. Nature. 2017;544:59-64.

92. Dekker J, Rippe K, Dekker M, Kleckner N. Capturing chromosome conformation. Science. 2002;295:1306-11.

93. Davison L, Wallace C, Cooper JD, Cope NF, Wilson NK, Smyth DJ, et al. Long-range DNA looping and gene expression analyses identify DEXI as an autoimmune disease candidate gene. Hum Mol Genet. 2012;21:322-33.

94. Zhao Z, Tavoosidana G, Sjölinder M, Göndör A, Mariano P, Wang S, et al. Circular chromosome conformation capture (4C) uncovers extensive networks of epigenetically regulated intra- and interchromosomal interactions. Nat Genet. 2006;38:1341-7.

95. Pasquali L, Gaulton KJ, Rodríguez-Seguí SA, Mularoni L, Miquel-Escalada I, Akerman I, et al. Pancreatic islet enhancer clusters enriched in type 2 diabetes risk-associated variants. Nat Genet. 2014;46:136-43.

96. Klein FA, Pakozdi T, Anders S, Ghavi-Helm Y, Furlong EEM, Huber W. FourCSeq: analysis of 4C sequencing data. Bioinformatics. 2015;31:3085-91.

97. Simonis M, Klous P, Homminga I, Galjaard R-J, Rijkers E-J, Grosveld F, et al. High-resolution identification of balanced and complex chromosomal rearrangements by 4C technology. Nat Methods. 2009;6:837-42.

98. Dostie J, Richmond TA, Arnaout RA, Selzer RR, Lee WL, Honan TA, et al. Chromosome Conformation Capture Carbon Copy (5C): a massively parallel solution for mapping interactions between genomic elements. Genome Res. 2006;16:1299-309.

99. Sanyal A, Lajoie BR, Jain G, Dekker J. The long-range interaction landscape of gene promoters. Nature. 2012;489:109-13.

100. Sauria ME, Phillips-Cremins JE, Corces VG, Taylor J. HiFive: a tool suite for easy and efficient $\mathrm{HiC}$ and 5C data analysis. Genome Biol. 2015;16:237.

101. Naumova N, Imakaev M, Fudenberg G, Zhan Y, Lajoie BR, Mirny LA, et al. Organization of the mitotic chromosome. Science. 2013;342:948-53.

102. Zhang Y, McCord RP, Ho Y-J, Lajoie BR, Hildebrand DG, Simon AC, et al. Spatial organization of the mouse genome and its role in recurrent chromosomal translocations. Cell. 2012;148:908-21.

103. Kalhor $\mathrm{R}$, Tjong $\mathrm{H}$, Jayathilaka N, Alber F, Chen L. Genome architectures revealed by tethered chromosome conformation capture and populationbased modeling. Nat Biotechnol. 2011;30:90-8.

104. Davies JOJ, Telenius JM, McGowan SJ, Roberts NA, Taylor S, Higgs DR, et al. Multiplexed analysis of chromosome conformation at vastly improved sensitivity. Nat Methods. 2016;13:74-80.

105. Platt JL, Salama R, Smythies J, Choudhry H, Davies JOJ, Hughes JR, et al. Capture- $C$ reveals preformed chromatin interactions between HIF-binding sites and distant promoters. EMBO Rep. 2016;17:1410-21.

106. de Vree PJP, de Wit E, Yilmaz M, van de Heijning M, Klous P, Verstegen MJAM, et al. Targeted sequencing by proximity ligation for comprehensive variant detection and local haplotyping. Nat Biotechnol. 2014;32:1019-25.

107. Hottentot QP, van Min M, Splinter E, White SJ. Targeted locus amplification and next-generation sequencing. Methods Mol Biol. 2017;1492:185-96. 
108. Kolovos P, van de Werken HJ, Kepper N, Zuin J, Brouwer RW, Kockx CE, et al. Targeted Chromatin Capture (T2C): a novel high resolution high throughput method to detect genomic interactions and regulatory elements. Epigenetics Chromatin. 2014;7:10

109. Cairns J, Freire-Pritchett P, Wingett SW, Várnai C, Dimond A, Plagnol V, et al. CHiCAGO: robust detection of DNA looping interactions in Capture Hi-C data. Genome Biol. 2016;17:127.

110. Nagano T, Lubling Y, Várnai C, Dudley C, Leung W, Baran Y, et al. Cell-cycle dynamics of chromosomal organization at single-cell resolution. Nature. 2017:547:61-7.

111. Flyamer IM, Gassler J, Imakaev M, Brandão HB, Ulianov SV, Abdennur N, et al. Single-nucleus $\mathrm{Hi}-\mathrm{C}$ reveals unique chromatin reorganization at oocyte-to-zygote transition. Nature. 2017:544:110-4.

112. Fullwood MJ, Liu MH, Pan YF, Liu J, Xu H, Mohamed YB, et al. An oestrogenreceptor-alpha-bound human chromatin interactome. Nature. 2009:462:58-64.

113. Li G, Chen Y, Snyder MP, Zhang MQ. ChIA-PET2: a versatile and flexible pipeline for ChIA-PET data analysis. Nucleic Acids Res. 2017;45:e4.

114. Servant N, Varoquaux N, Lajoie BR, Viara E, Chen C-J, Vert J-P, et al. HiC-Pro: an optimized and flexible pipeline for $\mathrm{Hi}-\mathrm{C}$ data processing. Genome Biol. 2015;16:259.

115. Ay F, Bailey TL, Noble WS. Statistical confidence estimation for Hi-C data reveals regulatory chromatin contacts. Genome Res. 2014;24:999-1011.

116. Phanstiel DH, Boyle AP, Heidari N, Snyder MP. Mango: a bias-correcting ChIA-PET analysis pipeline. Bioinformatics. 2015;31:3092-8.

117. Durand NC, Shamim MS, Machol I, Rao SSP, Huntley MH, Lander ES, et al. Juicer provides a one-click system for analyzing loop-resolution $\mathrm{Hi}-\mathrm{C}$ experiments. Cell Syst. 2016;3:95-8.

118. Zhang Y, Liu T, Meyer CA, Eeckhoute J, Johnson DS, Bernstein BE, et al. Model-based analysis of ChIP-Seq (MACS). Genome Biol. 2008;9:R137.

119. Victoria-Acosta G, Vazquez-Santillan K, Jimenez-Hernandez L, Muñoz-Galindo L, Maldonado V, Martinez-Ruiz GU, et al. Epigenetic silencing of the XAF1 gene is mediated by the loss of CTCF binding. Sci Rep. 2015;5:14838.

120. Flavahan WA, Drier Y, Liau BB, Gillespie SM, Venteicher AS, StemmerRachamimov AO, et al. Insulator dysfunction and oncogene activation in IDH mutant gliomas. Nature. 2016;529:110-4.

121. Nolen LD, Boyle S, Ansari M, Pritchard E, Bickmore WA. Regional chromatin decompaction in Cornelia de Lange syndrome associated with NIPBL disruption can be uncoupled from cohesin and CTCF. Hum Mol Genet. 2013;22:4180-93.

122. Lai F, Orom UA, Cesaroni M, Beringer M, Taatjes DJ, Blobel GA, et al. Activating RNAs associate with Mediator to enhance chromatin architecture and transcription. Nature. 2013;494:497-501.

123. Risheg H, Graham Jr JM, Clark RD, Rogers RC, Opitz JM, Moeschler JB, et al. A recurrent mutation in MED12 leading to R961W causes Opitz-Kaveggia syndrome. Nat Genet. 2007;39:451-3.

124. Eriksson M, Brown WT, Gordon LB, Glynn MW, Singer J, Scott L, et al. Recurrent de novo point mutations in lamin A cause Hutchinson-Gilford progeria syndrome. Nature. 2003;423:293-8.

125. Goldman RD, Shumaker DK, Erdos MR, Eriksson M, Goldman AE, Gordon LB, et al. Accumulation of mutant lamin A causes progressive changes in nuclear architecture in Hutchinson-Gilford progeria syndrome. Proc Natl Acad Sci U S A. 2004;101:8963-8.

126. McCord RP, Nazario-Toole A, Zhang H, Chines PS, Zhan Y, Erdos MR, et al. Correlated alterations in genome organization, histone methylation, and DNA-lamin A/C interactions in Hutchinson-Gilford progeria syndrome. Genome Res. 2013;23:260-9.

127. Xiang J-F, Yin Q-F, Chen T, Zhang Y, Zhang X-O, Wu Z, et al. Human colorectal cancer-specific CCAT1-L IncRNA regulates long-range chromatin interactions at the MYC locus. Cell Res. 2014;24:513-31.

128. Maass PG, Rump A, Schulz H, Stricker S, Schulze L, Platzer K, et al. A misplaced IncRNA causes brachydactyly in humans. J Clin Invest. 2012;122:3990-4002. 